\title{
Minimizing the cumulative burden of hypertension to reduce the risk of end-stage renal disease
}

\author{
Kazuo Kobayashi ${ }^{1,2}$
}

Received: 2 September 2021 / Revised: 9 September 2021 / Accepted: 10 September 2021 / Published online: 13 October 2021

(C) The Japanese Society of Hypertension 2021

Hypertension is one of the most common diseases significantly increasing the risk of cardiovascular disease and chronic kidney disease (CKD). The prevalence of hypertension among adults has increased from 650 million to 1.28 billion in the last 30 years, with $41 \%$ of female and $51 \%$ of male patients with hypertension being undiagnosed [1]. Moreover, only $23 \%$ of female and $18 \%$ of male patients with hypertension have well-controlled blood pressure (BP) [1]. Although hypertension is easily diagnosed via BP measurement and treated with various drugs at a low cost, many patients continue to have poorly controlled hypertension. In Japan, of the 43 million patients with hypertension, 14 million are unaware of their hypertension, and 31 million have poorly controlled BP [2]. Hypertension is defined as BP of $140 / 90 \mathrm{mmHg}$ or higher. The Systolic Blood Pressure Intervention Trial reported that lower BP control is related to more favorable cardiovascular outcomes [3]. Thus, strict BP management is recommended for high-risk hypertensive patients. According to the Japanese Society of Hypertension Guidelines for the Management of Hypertension 2019 (JSH2019) [2], BP of less than 120/80 $\mathrm{mmHg}$ is considered normal; patients with BP of $120-140$ / 80-90 $\mathrm{mmHg}$ are considered to have high-normal or elevated BP. With these changes in the definition of hypertension, general physicians (GPs) should aim to adequately control BP in patients with hypertension, including those with "borderline" hypertension.

All Japanese adults have access to BP measurement through annual health checkups or to BP monitoring devices at

Kazuo Kobayashi

k-taishi@xc4.so-net.ne.jp

1 Committee of Hypertension and Kidney Disease, Kanagawa Physicians Association, Yokohama, Japan

2 Department of Medical Science and Cardiorenal Medicine, Yokohama City University Graduate School of Medicine, Kanagawa, Japan home. Although hypertension is sometimes detected when patients develop cardiovascular or kidney disease, patients with hypertension are frequently diagnosed during annual health checkups or by home BP monitoring.

Kim CS et al. concluded that the risk of end-stage renal disease (ESRD) increases to 1.35, 1.54, 1.51, and 2.28 among patients with a hypertension burden of 1, 2, 3, and 4, respectively, and the risk is more pronounced in patients with CKD (Fig. 1). Therefore, hypertension detection is related to the risk of ESRD. Thus, GPs should recognize ESRD risk in patients with hypertension, who should be treated immediately.

OMRON Corporation (Kyoto, Japan), a major company that manufactures BP measuring devices, conducted a survey among 10,000 Japanese people regarding their awareness of BP and hypertension [4]. Among the participants,

\section{The risk of for the end-stage renal disease by hypertension burden with or without CKD}

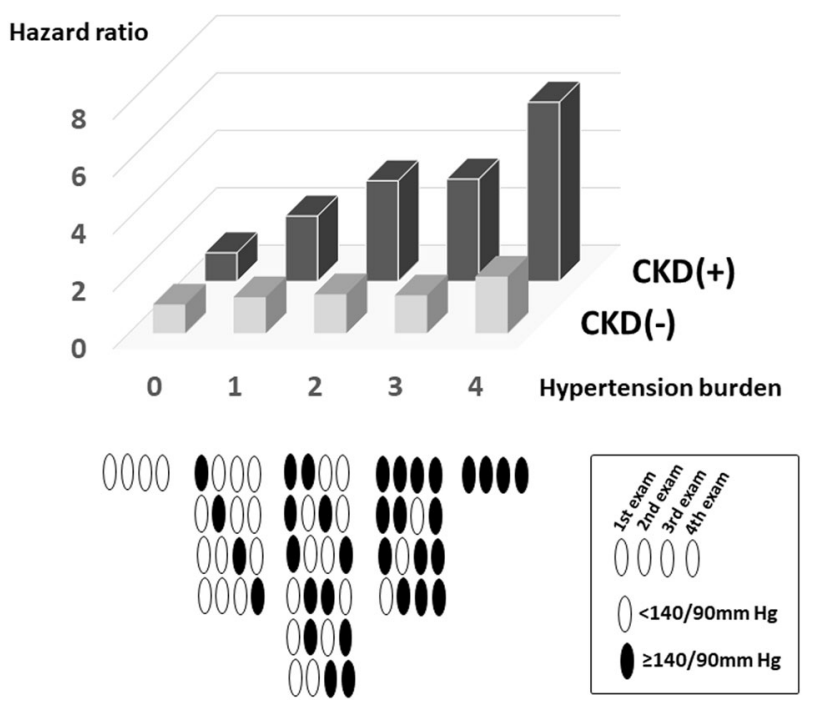

Fig. 1 The risk of end-stage renal disease by hypertension burden with or without CKD 
$32.1 \%$ had a high BP reading during their annual checkup. This rate became higher with age. Among them, 40.9\% restricted their salt intake, whereas $36.3 \%$ started monitoring their BP at home; however, only half of the participants with hypertension consulted a GP. More than $80 \%$ of the participants were unaware that $\mathrm{BP}$ can fluctuate during the day, and $60.4 \%$ did not know that hypertension can manifest asymptomatically. Many asymptomatic patients may think that their BP readings are only incidental and may not feel the need to consult a doctor, whereas others may refuse to consult a doctor, assuming that salt restriction alone is a sufficient control measure for hypertension. These passive attitudes regarding high BP contribute to neglected hypertension and an increased risk of cardiovascular or renal outcomes. The gap between the diagnosis and treatment of hypertension is a critical concern in the control of $\mathrm{BP}$ among patients with hypertension.

The notable novelties of this study are as follows. First, they clarify the relationship between BP and renal outcomes. Second, they establish the new concept of the "cumulative burden of hypertension", which is different from BP levels.

Until now, evidence revealing the relationship between $\mathrm{BP}$ and renal outcomes was less strong than that for cerebrovascular and cardiovascular outcomes. Kanno et al. reported adjusted hazard risks for the incidence of CKD in patients with prehypertension (120-139/80-89 mmHg), level I hypertension (140-159/90-99 mmHg), and level II hypertension $(\geq 160 / 100 \mathrm{mmHg})$ of $1.49,1.83$, and 2.55 , respectively, with a mean follow-up of 6.5 years, compared with that in patients with normal BP $(<120 / 80 \mathrm{mmHg})$ [5]. This novel study by Kim CS et al. clarifies the stratified risk for ESRD depending on the cumulative burden of hypertension. In this report by Kim CS et al., the risk rate of ESRD in patients in each group with a hypertension burden of $1,2,3$, and 4 was $1.35,1.54,1.51$, and 2.28 , respectively, compared with that in patients in a group with a hypertension burden of 0 . Their average BP (systolic/diastolic) levels were $127.91 \pm 11.66 / 81.2 \pm 8.62, \quad 134.48 \pm 12.24$ / $85.55 \pm 9.02, \quad 140.73 \pm 12.78 / 89.53 \pm 9.32$, and $149.15 \pm$ $12.38 / 94.73 \pm 8.74 \mathrm{mmHg}$, respectively. Due to the different endpoints, a direct comparison could not be performed; however, the burden of hypertension may be related to the risk for ESRD and BP progression. Previous evidence has mostly been generated from the viewpoint of BP level and outcomes; however, this study by Kim CS et al. has the novelty that the risk for renal outcomes was clarified by focusing on the burden of hypertension, which is different from the BP level. In JSH2019, the timing of initiating antihypertensive agents depended on the level of cardiovascular disease risk, as determined by BP levels and risk factors affecting prognosis (age $>65$ years, male sex, smoking, dyslipidemia, diabetes mellitus, brain hemorrhage, brain infarction, myocardial infarction, nonvalvular atrial fibrillation, and proteinuria) [2]. Based on the results from this study, the cumulative burden of hypertension was considered a new risk factor affecting the prognosis of kidney disease.

Overall, it is imperative to minimize the burden of hypertension to prevent kidney damage. As early treatment of hypertension effectively reduces this risk for ESRD, GPs (whom many patients with hypertension may first consult) should promote early intervention, including salt restriction and medication. The mindset for the treatment of hypertension should be geared toward early treatment. Moreover, a prospective study should be conducted to evaluate this strategy.

Kim CS et al. also aimed to analyze a large and reliable national Korean database. The results from the survey in Japan based on the J-CKD database have already been reported [6-8]. The database of the association of clinical medicine (J-DOME) is currently under investigation in Japan. New evidence related to renal outcomes based on these databases should be created in the future.

\section{Compliance with ethical standards}

Conflict of interest The author declares no competing interests.

Publisher's note Springer Nature remains neutral with regard to jurisdictional claims in published maps and institutional affiliations.

\section{References}

1. Zhou B, Carrillo-Larco RM, Danaei G, Riley LM, Paciorek CJ, Stevens GA, et al. Worldwide trends in hypertension prevalence and progress in treatment and control from 1990 to 2019: a pooled analysis of 1201 population-representative studies with 104 million participants. The Lancet. https://doi.org/10.1016/s0140-6736(21) 01330-1 (2021).

2. Umemura S, Arima H, Arima S, Asayama K, Dohi Y, Hirooka Y, et al. The Japanese Society of Hypertension Guidelines for the Management of Hypertension (JSH 2019). Hypertension Res. 2019;42:1235-481.

3. Group TSR. A randomized trial of intensive versus standard bloodpressure control. N. Engl J Med. 2015;373:2103-16.

4. cooporate $\mathrm{O}$ https://www.healthcareomroncojp/corp/news/pdf/ 20170511pdf.

5. Kanno A, Kikuya M, Ohkubo T, Hashimoto T, Satoh M, Hirose T, et al. Pre-hypertension as a significant predictor of chronic kidney disease in a general population: the Ohasama Study. Nephrol Dialysis Transplant. 2012;27:3218-23.

6. Sofue T, Nakagawa N, Kanda E, Nagasu H, Matsushita K, Nangaku M, et al. Prevalences of hyperuricemia and electrolyte abnormalities in patients with chronic kidney disease in Japan: a nationwide, cross-sectional cohort study using data from the Japan Chronic Kidney Disease Database (J-CKD-DB). PLoS ONE. 2020;15:e0240402.

7. Sofue T, Nakagawa N, Kanda E, Nagasu H, Matsushita K, Nangaku M, et al. Prevalence of anemia in patients with chronic kidney disease in Japan: A nationwide, cross-sectional cohort study using 
data from the Japan Chronic Kidney Disease Database (J-CKDDB). PLoS ONE. 2020;15:e0236132.

8. Nakagawa N, Sofue T, Kanda E, Nagasu H, Matsushita K, Nangaku M, et al. J-CKD-DB: a nationwide multicentre electronic health record-based chronic kidney disease database in Japan. Sci Rep. 2020;10:7351. 